\title{
ON THE ANALYTIC COHOMOLOGY OF A DOMAIN IN A STEIN MANIFOLD
}

\author{
MICHAEL G. EASTWOOD
}

\begin{abstract}
Suppose $M$ is an open subset of a Stein manifold without isolated points and that $V$ is a holomorphic vector-bundle on $M$ admitting a holomorphic connection. Then $H^{q}(M, \theta(V))$ is either zero or of infinite dimension.
\end{abstract}

In [1] Laufer showed that, for $M$ an open subset of a Stein manifold without isolated points, $H^{q}\left(M, \Omega^{p}\right)$ is either zero or of infinite dimension as a complex vector-space. Here $\Omega^{p}$ denotes the sheaf of germs of holomorphic $p$-forms. In this note we extend this result to $H^{q}(M, \vartheta(V))$ for any holomorphic vector-bundle $V$ obtained as the restriction of a holomorphic vector-bundle on the ambient Stein manifold (see corollary below). The argument is an extension of that given by Laufer for $H^{q}(M, \theta)$. The crucial ingredient is a connection on $V$ (see theorem below).

Since making this extension I have learned that these results were also obtained by John Sensat, a student of Professor Laufer. Unfortunately, he was killed in an accident approximately three years ago. I respectfully dedicate this paper to his memory.

Lemma. Suppose $V$ is a holomorphic vector-bundle on a complex manifold $M$. Suppose $V$ admits a holomorphic connection. Fix $a$ nonnegative integer $q$ and let $J$ denote the ideal of holomorphic functions on $M$ which induce (by multiplication on the sheaf level) the zero map of $H^{q}(M, \mathcal{O}(V))$ to itself. Then $f \in J \Rightarrow X f \in J$ for any holomorphic vector-field $X$ on $M$.

Proof. Let $\nabla: \theta(V) \rightarrow \Omega^{1}(V)$ be a connection. By the Leibnitz rule $(X f) s$ $=\nabla_{X}(f s)-f \nabla_{X} s$ for $s \in \mathcal{O}(V)$. By functoriality the same is true for $s \in$ $H^{q}(M, \Theta(V))$ and the result follows.

THEOREM. Suppose $M$ is an open subset of a Stein manifold without isolated points. Suppose $V$ is a holomorphic vector-bundle on $M$ that admits a holomorphic connection. Then $H^{q}(M, \vartheta(V))$ is either zero or of infinite dimension.

Proof. Denote by $S$ the ambient Stein manifold and regard $S$ as being embedded in $\mathbf{C}^{N}$. Let $I$ denote the ideal of holomorphic functions on $S$ which induce the zero map on $H^{q}(M, \theta(V))$, i.e., using the notation of the lemma above, $\left.f \in I \Leftrightarrow f\right|_{M} \in J$. Suppose $H^{q}(M, \vartheta(V))$ is of finite dimension. It suffices to show

Received by the editors November 15, 1980.

1980 Mathematics Subject Classification. Primary 32C35; Secondary 32C10.

(c) 1981 American Mathematical Society $0002-9939 / 81 / 0000-0421 / \$ 01.50$ 
that $1 \in I$. The coordinate functions $z_{j}$ on $\mathbf{C}^{N}$ induce linear endomorphisms of $H^{q}(M, \vartheta(V))$. Let $p_{j}$ be the minimal polynomial of $z_{j}$ regarded as such an endomorphism. Then $p_{j}\left(z_{j}\right) \in I$ and the collection $p_{1}\left(z_{1}\right), \ldots, p_{N}\left(z_{N}\right)$ have only finitely many common zeros on $S$. However, for any $x \in S$ we can choose $f \in I$ with $f(x) \neq 0$, for first choose any nonzero $f \in I$ and then use the lemma above repeatedly to reduce the order of the zero. Here we are using Cartan's Theorem B to assert that we can specify the value at $x$ of a holomorphic vector-field on $S$. Thus we can augment our collection $p_{j}\left(z_{j}\right)$ to a collection, say $f_{1}, \ldots, f_{n} \in I$, with no common zeros. Now Theorem B shows that we can find $g_{1}, \ldots, g_{n} \in \Gamma(S, \theta)$ with $\sum g_{j} f_{j}=1$ and so $l \in I$ as required.

Corollary. Suppose $M$ is an open subset of a Stein manifold $S$ without isolated points. Suppose $V$ is a holomorphic vector-bundle on $S$. Then $H^{q}(M, \vartheta(V))$ is either zero or of infinite dimension.

Proof. $V$ admits a holomorphic connection on $S$ since the obstruction lies in $H^{1}\left(S, \Omega^{1}(\right.$ End $\left.V)\right)$ and this vanishes by Cartan's Theorem B. Now restrict to $M$ and apply the theorem.

\section{REFERENCES}

1. H. B. Laufer, On the infinite dimensionality of the Dolbeault cohomology groups, Proc. Amer. Math. Soc. 52 (1975), 293-296.

School of Mathematics, Institute for Advanced Study, Princeton, New Jersey 08540

Current address: Mathematical Institute, 24-29 St. Giles', Oxford, OX1 3LB, England 